\title{
$\widehat{A}$ Madridge \\ madridge Journal of Otorhinolaryngology \\ Interconnecting Scientific World
}

Research Article

Open Access

\section{Lipid Profile in Patients with Sensorineural Hearing Loss - One Year Observational Study in a Tertiary Care Centre}

\author{
RS Mudhol and Amreen Patwegar \\ Department of ENT and HNS, Jawaharlal Nehru Medical College, KAHER, Belagavi, Karnataka, India
}

\section{Article Info}

*Corresponding author:
Amreen Patwegar
Department of ENT and HNS
Jawaharlal Nehru Medical College
Nehru Nagar
Belagavi, Karnataka
India
E-mail: amreen.patwegar@gmail.com

Received: September 24, 2019

Accepted: October 4, 2019

Published: October 11, 2019

Citation: Mudhol RS, Patwegar A. Lipid Profile in Patients with Sensorineural Hearing Loss - One Year Observational Study in a Tertiary Care Centre. Madridge J Otorhinolaryngol. 2019; 4(1): 77-80. doi: $10.18689 / \mathrm{mjol}-1000116$

Copyright: (c) 2019 The Author(s). This work is licensed under a Creative Commons Attribution 4.0 International License, which permits unrestricted use, distribution, and reproduction in any medium, provided the original work is properly cited.

Published by Madridge Publishers

\begin{abstract}
Background: The negative influence of hypercholesterolemia on the blood vessels' condition and following degeneration lesions of the organs is well known. There have been several studies which previously established a relation between raised lipid levels and hearing disorders, but till date the theory remains questionable.
\end{abstract}

Objective: To study association among various degrees of sensorineural hearing loss and serum levels of total cholesterol, triglycerides, HDL (high density lipoprotein) and LDL (low density lipoprotein).

\section{Study design: An observational study}

Methods: In all, 58 cases between 18 and 60 years with sensorineural hearing loss, during the study period of 1 year from Jan 2018 to Dec 2018 were selected and investigated by pure tone audiometry for assessing the hearing loss and their fasting blood samples investigated for lipid levels. Sensorineural hearing loss was classified as mild, moderate, moderately severe, severe and profound based on the pure tone average of the worse hearing ear.

Results: Serum levels of high density cholesterol and triglycerides were found to have a negative correlation with hearing loss (-1.46 and -1.52$)$. Their $p$ values were 0.1488 and 0.1329 . Similarly, the low density cholesterol and total cholesterol levels had a minimal positive correlation with the hearing loss (0.99 and 0.46$)$, and their $p$ values are 0.3260 and 0.6435 each.

Conclusion: The serum levels of low density lipoprotein directly correlate with the severity of sensorineural hearing loss whereas the serum levels of high density lipoprotein negatively correlate with the severity of sensorineural hearing loss in both sexes, but not significantly.

Keywords: Sensorineural hearing loss; Serum lipids; Total Cholesterol; Triglycerides; Low density lipoproteins; High density lipoproteins.

Abbreviations: $d B$ : Decibel, unit of sound intensity; HDL: High density lipoprotein: LDL: Low density lipoprotein; HLOSS: Hearing loss; PTA: Pure tone audiometry; SNHL: Sensorineural hearing loss; T.C: Total Cholesterol; TRIGLYDS: Triglycerides.

\section{Introduction}

Hearing loss is a frequent observation in the general population with many etiologies. The normal physiological mechanisms of the human body face new challenges everyday due to the modern life style. The adverse impact of this modern, sedentary lifestyle, excess body weight and a diet rich in total and saturated fats, superimposed on 
a genetic background confers susceptibility to increased circulating lipids. The negative influence of hypercholesterolemia on the blood vessels' condition and following degenerative lesions of the organs is well known [1].

The various mechanisms by which hyperlipidemia might cause SNHL have been postulated and these include atherosclerosis, lipidosis, metabolic, micro vascular disease, hypoxia, embolic episode, hyper viscosity, hereditary, ageing and hypertension [2].

In recent years, cochlear ischemia has been hypothesized in patients of sensorineural hearing loss, in whom an infectious episode or acoustic neuroma have been excluded [3]. Estimation of serum lipids and their correlation with multiple variants and levels of SNHL may provide an insight into this relationship. Suzuki et al. [3] in their study found hearing levels at $2000 \mathrm{~Hz}$ and $4000 \mathrm{~Hz}$ for group with high HDL cholesterol were significantly better than those in the low $\mathrm{HDL}$ cholesterol group in men.

The higher incidence of hearing loss among patients with hyperlipidemia has long been established and is constantly being verified by new studies.

This study was designed to emphasize the need for early detection of hyperlipidemia in SNHL patients who were otherwise not under the care of physicians. And in doing so, a metabolic abnormality which apparently leads to high risks for coronary artery disease, hearing loss, vestibular dysfunction and other illnesses may be better controlled.

\section{Materials and Methods}

\section{Study duration and source of data}

Patients aged between 18 and 60 years with sensorineural hearing loss attending the ENT \& HNS outpatient department in KLES Dr Prabhakar Kore Hospital, Belagavi, India were studied. A total of 58 patients during the study period of one year from January 2018 to December 2018 were included.

\section{Exclusion criteria}

Patients with chronic suppurative otitis media, diabetes mellitus, chronic alcoholism, myxedema, nephrotic syndrome, and those on drugs affecting hearing and lipid levels like statins, oral contraceptives and beta-blockers were excluded.

Also patients with sensorineural hearing loss with a known cause like Meniere's disease, labyrinthitis, acoustic neuroma, temporal bone fracture, syphilis, meningitis and noise induced hearing loss were excluded.

\section{Study design}

An observational study.

\section{Ethical Clearance}

The study was approved by the institutional ethical committee. The research was approved as per the letter no -MDC/DOME/74. Written and informed consent was taken from all patients.

\section{Methodology}

The patients' detailed history was obtained for duration of hearing loss, duration and nature of any previous treatment. All patients were clinically examined including general physical examination, detailed ENT examination and otoscopic examination of the ear. Tuning fork tests (Rinne's Test, Weber's Test, and Absolute Bone Conduction) were performed.

Pure tone audiometry was performed on all patients to evaluate hearing profile. The degree of sensorineural hearing loss was based on the WHO classification and classified as shown in table $1[4,5]$.

Table 1. WHO classification of Degree of hearing loss.

\begin{tabular}{|c|l|c|}
\hline & Degree of hearing loss & Hearing threshold (Pure tone average) \\
\hline 1 & Mild & $26-40 \mathrm{~dB}$ \\
\hline 2 & Moderate & $41-55 \mathrm{~dB}$ \\
\hline 3 & Moderately Severe & $56-70 \mathrm{~dB}$ \\
\hline 4 & Severe & $71-90 \mathrm{~dB}$ \\
\hline 5 & Profound & $>91 \mathrm{~dB}$ \\
\hline
\end{tabular}

The fasting serum lipid profile estimation was done by using a commercial kit available for:

- HDL

- LDL

- Triglycerides

- Total cholesterol.

Triglycerides, total cholesterol, HDL were the measured parameters; LDL was calculated by an indirect method using the Friedewald's formula.

$L D L=$ Total Cholesterol $-\left(H D L+\frac{\text { IRIGLYCERIDE }}{\mathbf{5}}\right)$

\section{Statistical Analysis}

Data was analyzed by:

1. Mean \pm Standard deviation.

2. Chi square test.

3. Kolmogorov Smirnov test for normalcy of the parameters.

4. Karl Pearson's correlation coefficient method to check the correlation.

\section{Results}

Out of 58 cases, 4 (7\%) patients were having minimal hearing loss, 16 (28\%) patients were having mild SNHL, 12 (21\%) patients were having moderate SNHL, 9 (15\%) patients were having moderately severe SNHL, 15 (26\%) were having severe SNHL and 2 (3\%) patients were having profound SNHL. The male:female ratio in the study population was 1.15:1.

\section{Distribution of males and females by age groups}

The distribution according to the age groups show that, 10 males and 2 females are in ages $\leq 30$ yrs. 6 males and 6 females are between 31-40 yrs, 3 males and 8 females are between 41-50 yrs, 12 males and 11 females are between 51$60 \mathrm{yrs}$. The $\mathrm{p}$ value was $=0.0601$, which showed that a direct relationship exists between the age and severity of hearing loss (Figure 1). 


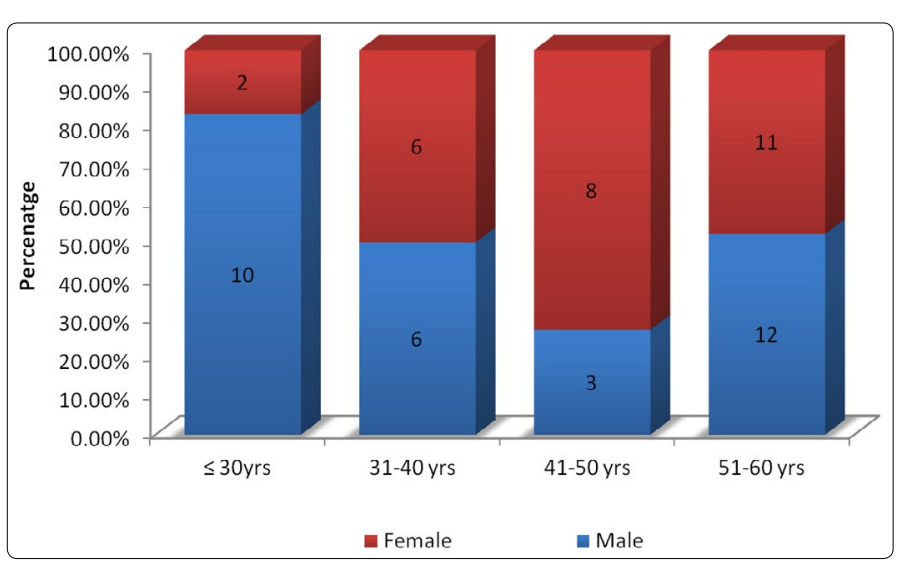

Figure 1. Distribution of males and females by age groups.

\section{Correlation between age in yrs with lipid parameters}

The 58 patients were categorized into 4 groups according to their ages, and the correlation between age and lipid profile was done. It was found that, HDL and triglycerides had a strong negative correlation (-2.02 and -2.67$)$ with advancing age, and it was also significant. The $p$ values were 0.047 for HDL and 0.0099 for Triglycerides.

Total Cholesterol showed a negative correlation with advancing age $(-0.925)$, with $p$ value of 0.35 and $L D L$ showed a positive correlation with age $(+0.548)$ with a $p$ value of 0.58 (Figure 2 ).

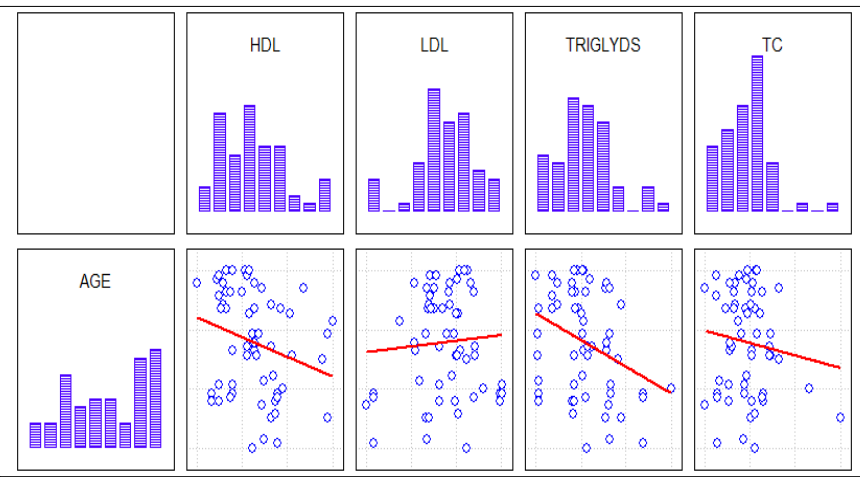

Figure 2. Scatter plot of correlation between age and lipid parameters.

\section{Correlation between hearing loss and lipid parameters}

It was found that HDL and Triglycerides were having a negative correlation with increasing degree of hearing loss (-1.46 and -1.52 ). Their $p$ values were 0.1488 and 0.1329 . Similarly, the LDL and Total Cholesterol levels had a minimal positive correlation with the increasing degree of hearing loss (0.99 and 0.46 ), and their $p$ values are 0.3260 and 0.6435 each (Figure 3 ).

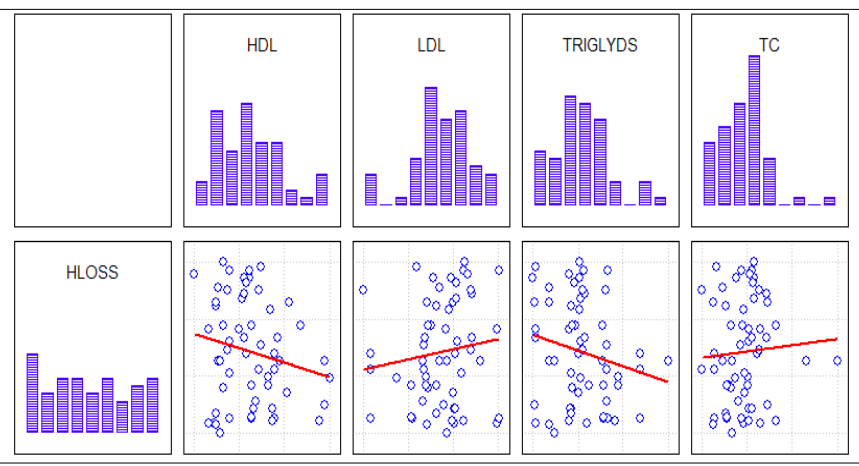

Figure 3. Scatter plot of correlation between hearing loss and lipid parameters.

\section{Discussion}

The results of this study show that there are significant alterations in the lipid profiles among different degrees of sensorineural hearing loss.

All continuous variables are presented as the mean \pm standard deviation and compared using independent $t$ test. Categorical data are shown as percentages and compared using the Chi square test.

Anil and Shazia [6] in 2016 studied 100 adults aged 30-60 years with SNHL. Their study showed significant relationship between serum lipid and various degrees of sensorineural hearing loss. Increase in total cholesterol, triglycerides and $L D L$, were associated with worsening of hearing levels.

A study conducted by Jalisatgi [2] in 2009 in Bijapur, India on 64 patients with SNHL showed that serum levels of LDL directly correlate with the severity of sensorineural hearing loss whereas HDL levels correlate negatively.

\section{Comparison of LDL in the whole study group}

Lee et al. [7] found in their study the mean LDL levels to be $185.33 \pm 20.32$, whereas in the present study the mean LDL value was found to be $108.1 \pm 26.7$. The levels of serum LDL had a positive correlation with the hearing levels in both males and female populations of the study group (Table 2).

Table 2. Comparison of LDL in the whole study group.

\begin{tabular}{|l|c|}
\hline Studies & Mean LDL(mg/dl) \\
\hline Lee FS et al. [7] & $185.33 \pm 20.32$ \\
\hline Present study & $108.1 \pm 26.7$ \\
\hline
\end{tabular}

\section{Comparison of HDL in the whole study group}

Lee et al. [7] in their study found the mean HDL values to be $16.00 \pm 6.43 \mathrm{mg} / \mathrm{dl}$. In the present study, the mean HDL values were found to be $48.77 \pm 13.08 \mathrm{mg} / \mathrm{dl}$, and these values had a significant negative correlation with advancing age $(p=0.047)$ (Table 3$)$.

Table 3. Comparison of HDL in the whole study group

\begin{tabular}{|l|c|}
\hline Studies & Mean HDL(mg/dl) \\
\hline Lee FS et al. [7] & $16 \pm 6.43$ \\
\hline Present study & $48.77 \pm 13.08$ \\
\hline
\end{tabular}

In comparison with NCEP Adult Treatment Panel III guidelines $[8,9]$ the total cholesterol is within normal limits among different degrees of hearing loss. The LDL levels were found to be near optimal. The HDL and triglyceride levels were found to be high in case of mild and moderate hearing loss, low in cases of moderately severe and severe hearing loss.

Friedrich et al. [10] in 1981 found no change in serum triglyceride or total cholesterol concentrations in 49 patients with neuro-otological symptoms, but these patients had significantly elevated $L D L$ and ratio of $L D L / H D L$ in serum. Hence the study suggested an association between atherosclerosis and inner ear dysfunction.

Gates et al. [11] reported no correlation of hearing levels with total cholesterol or triglycerides, but HDL in women negatively correlated with hearing levels. 
In the present study HDL and triglycerides negatively correlated with hearing levels in both males and females.

A study by Ullrich et al. [12] indicated that both hyperlipidaemia and atherogenic risk factors do not have major pathological importance in sudden SNHL.

Also, Kazmierczak and Doroszeweska [13] concluded that the disturbance of glucose metabolism and hyperinsulinemia may be responsible for inner ear diseases, whereas role of disturbance of lipid metabolism remains vague.

Likewise, Rudack et al. [14] found correlation between HDL-CH and LDL-CH and sudden SNHL.

These results suggests that a high serum LDL and low $\mathrm{HDL}$ is associated with atherosclerosis-related micro circulatory disturbances of the cochlear vasculature and also increased susceptibility of the cochlea to noise. When combined with daily exposure to noise, these changes can lead to hearing loss. Anti-lipid therapy and low cholesterol diet can provide significant improvement by way of lowered tinnitus intensity and improvement in average hearing thresholds.

\section{Conclusion}

Auditory disturbances in the general population and its association with serum lipids have been studied clinically and experimentally, but their relation remains controversial.

In this study, alteration in different lipoprotein fractions in various degrees of sensorineural hearing loss was studied. From the observations in the study, we can conclude that there is a need for continual assessment of serum lipid levels in people with sensorineural hearing loss and it should be managed effectively to prevent the further progression of sensorineural hearing loss.

\section{Conflict of Interest}

Authors state no conflict of interest.

\section{References}

1. Mohammed AA. Lipid profile among patients with sudden sensorineural hearing loss. Indian J Otolaryngol Head Neck Surg. 2014; 66(4): 425-428. doi: 10.1007/s12070-014-0744-0

2. Jalisatgi RR, Guggarigoudar SP. A study of relationship between serum lipid levels and sensorineural hearing loss. RGUHS Dissertation. 2009: 1415.

3. Suzuki K, Kaneko M, Murai K. Influence of Serum Lipids on auditory function. Laryngoscope. 2000; 110: 1736-1738. doi: 10.1097/00005537200010000-00033

4. Wright A. Anatomy and ultrastructure of the human ear. Scott Browns Otolaryngology, Basic sciences. 6 $^{\text {th }}$ edition. Butterworth London; 1997.

5. Gale J, Forge A. Anatomy of the cochlea and vestibular system: Relating ultrastructure to function. In: Watkinson JC, Clarke RW (eds). ScottBrown's Otolaryngology and Head and Neck Surgery. $8^{\text {th }}$ edition. CRC Press; 2018.

6. Anil HT, Shazia. A Study of Relationship between Serum Lipids and Sensorineural Hearing Loss. JMSCR. 2016; 4(7): 11570-11573. doi: 10.18535/jmscr/v4i7.65

7. Lee FS, Matthews $L$, Mills JH, Dubno JR, Adkins WY. Analysis of blood chemistry and hearing levels in a sample of older persons. Ear Hear. 1998; 19(3): 180-190. doi: 10.1097/00003446-199806000-00002

8. Expert Panel on Detection, Evaluation, and Treatment of High Blood Cholesterol in Adults. Executive summary of the third report of the National Cholesterol Education Program (NCEP) expert panel on detection, evaluation, and treatment of high blood cholesterol in adults (adult treatment panel III). JAMA. 2001; 285(19): 2486-2497. doi: 10.1001/ jama.285.19.2486

9. Grundy SM, Cleeman Jl, Merz CN, et al. Implications of recent clinical trials for National Cholesterol Education Program Adult Treatment Panel III Guidelines. Circulation. 2004; 110(2): 227-239. doi: 10.1161/01.CIR.0000133317.49796.0E

10. Friederich G, Pilger E. Lipoproteins in cochleovestibular disorders. Arch Otorhinolaryngol. 1981; 232(2): 101-105. doi: 10.1007/bf00505028

11. Gates GA, Cobb JL, D'Agostino RB, Wolf PA. The relation of hearing in the elderly to the presence of cardiovascular disease and cardiovascular risk factors. Arch Otolaryngol Head Neck Surg. 1993; 119(2): 156-161. doi: 10.1001/archotol.1993.01880140038006

12. Ullrich $D$, Aurbach $G$, Drobik C. A prospective study of hyperlipidaemia as a pathogenic factor in sudden sensor neural hearing loss. Eur Arch Otorhinolaryngol. 1992; 249(5): 273-276. doi: 10.1007/BF00714491

13. Kazmierczak $H$, Doroszeweska $G$. Metabolic disorders in vertigo, tinnitus, and hearing loss. Int Tinnitus J. 2001; 7(1): 54-58.

14. Rudack $C$, Langer $C$, Stoll W, Rust $S$, Walter $M$. Vascular risk factors in sudden hearing loss. Thromb Haemost. 2006; 95(3): 454-461. doi: 10.1160/ TH05-08-0554 